\title{
Nephrotic Syndrome Due to Primary Renal Disease in Adults: I. Survey of Incidence in South-east England
}

\author{
P. SHARPSTONE,* M.B., M.R.C.P. ; C. S. OGG, † M.D., B.SC., M.R.C.P. ; J. S. CAMERON, $\ddagger$ M.D., B.SC., M.R.C.P.
}

\begin{abstract}
Cummary : In a prospective survey of the nephrotic syndrome due to primary renal disease in adult patients in the South-east Metropolitan Region of England 50 patients were seen in a two-year period-a minimum annual incidence of $\mathbf{9 . 0}$ new cases per million adult population. The frequency distribution of the three main histological groups was "minimal change" $\mathbf{3 0} \%$, membranous nephropathy $12 \%$, and proliferative glomerulonephritis $58 \%$. The higher proportion of patients with minimal histological change compared with that found in most previously published series may be explained by the avoidance of selection of patients for inclusion. The much lower incidence of membranous nephropathy probably reflects the use of stricter histological criteria for this diagnosis.
\end{abstract}

\section{Introduction}

The incidence of the nephrotic syndrome in adults has never been assessed and the frequency distribution of the different histological patterns seen on renal biopsy varies widely in published series (Robson, 1967). Some of this variation must depend on the method of selection of the patients, and in particular these series may have been weighted by the inclusion of a high proportion of patients with more severe or intractable disease. Such patients are more of ten referred to the specialized renal centres from which the series have been reported.

In the present investigation, which is concerned with adults only, an attempt was made to survey as high a proportion as possible of new cases of the nephrotic syndrome (defined by arbitrary but strict criteria) in the south-east of England. To avoid bias in selection so far as was possible a prospective study was carried out limited to patients who had had neither previous treatment nor renal biopsy. Patients with the nephrotic syndrome associated with multi-system disease, such as diabetes, systemic lupus erythematosus, and amyloidosis, were not included in the survey, since here the pathological diagnosis is usually made without recourse to renal biopsy, and the possible reluctance of some physicians to refer these patients for biopsy would have introduced an element of bias.

\section{Methods}

The survey covered the two-year period from 1 July 1966 to 30 June 1968 and embraced the areas of South-east London, Kent, Sussex, and Surrey served by the South-east Metropolitan Regional Hospital Board. At the beginning of this period a circular was sent, with the co-operation of the regional board, to all consultant general physicians under contract with the board outlining the proposed survey and the associated therapeutic trial (Sharpstone et al., 1969). They were invited to notify us of patients fulfilling the following criteria: (1) proteinuria greater than $3 \mathrm{~g} . / 24$ hours (averaged over three

* Lecturer in Medicine, King's College Hospital Medical School, London S.E.5.

† Renal Physician, Guy's Hospital, London S.E.1.

$¥$ Renal Physician and Senior Lectures, Department of Medicine, Guy's FIospital, London S.E.1. days), (2) serum albumin less than $3 \mathrm{~g} . / 100 \mathrm{ml}$., (3) oedema at the time of or within two weeks of referral, (4) aged over 14 years, (5) no previous treatment with corticosteroid or other immunosuppressive drug, and (6) the absence of evidence of any multi-system disease with which the nephrotic syndrome may be associated (except Henoch-Schönlein purpura). The patients remained at all times under the care of the referring physician and renal biopsy was performed by one of us at the referring hospital. Chemical estimations on aliquots of three consecutive 24-hour urine collections and plasma samples from each patient were carried out centrally to ensure that the biochemical criteria for inclusion in the survey were comparable for all patients.

Serum albumin was estimated on an AutoAnalyzer by the biuret method of Hiller et al. (1927) with ammonium sulphate precipitation of globulins. Urine protein was measured by the biuret method of Hiller et al. (1927). Renal biopsy was performed by a modification of the technique described by Kark and Muehrcke (1954) and using the Franklin modified VimSilverman biopsy needles and local analgesia.

\section{Assessment of Renal Histology}

Specimens were fixed in $10 \%$ buffered formalin, though in some later biopsies alcoholic picrate was used. The fixed tissue was embedded in paraffin, cut at $3 \mu$, and examined by optical microscopy with the use of haemalum-eosin, periodic-acidSchiff, and periodic-acid-silver-methenamine stains. Amyloid was detected by Congo red staining and examination with polarized light, and by thioflavine $\mathrm{T}$ staining with examination under ultraviolet light.

Specimens were allocated to the following groups: (1) minimal change, (2) membranous nephropathy, and (3) proliferative glomerulonephritis. Five subgroups of No. 3 were sought: (a) acute diffuse/exudative, (b) lobular stalk thickening, (c) membranoproliferative, (d) with extensive crescents, and $(e)$ all other proliferative glomerulonephritis. Details of the criteria used in the allocation have been described elsewhere (Ogg et al., 1968).

In the present unsatisfactory state of our knowledge of proliferative glomerulonephritis group $3(e)$ embraces a variety of appearances, including patients with mainly endothelial proliferative glomerulonephritis and having a variable degree of glomerulosclerosis and deposition of silver- and P.A.S.-positive fibrillar material. This material and the excess of endothelial cells have no particular distribution within the tuft, but sometimes affect one area or lobule of a glomerulus. Also included in this group are a few patients with advanced glomerulosclerosis and endothelial proliferation whose original type of proliferative glomerulonephritis is in doubt.

\section{Results}

In the two-year period of the survey 56 patients aged 14 years and over who had the nephrotic syndrome apparently due to primary renal disease were seen in the South-east Metropolitan Region. Renal biopsies from these 56 patients 
showed that six had unsuspected amyloidosis. The population aged 14 and over from which the patients were drawn numbered $2,770,000$, so that the 50 new patients represent a minimal annual incidence of the nephrotic syndrome due to primary renal disease of 9.0 per million adults. Twenty-eight patients were female and 22 male (Table I). Twenty-seven were seen in the first year and 23 in the second year of the survey, indicating that there was no important inflation of the incidence figure by the referral of old patients at the time of starting the survey.

TABLE I. - Sex Distribution

\begin{tabular}{llll|c|c|c}
\hline & & & & Female & Male & Ratio \\
\hline Minimal change $\ldots$ & $\ldots$ & $\ldots$ & $\ldots$ & 11 & 4 & $\begin{array}{l}2.75: 1 \\
\text { Other histology }\end{array}$. \\
\hline
\end{tabular}

Renal biopsy provided an adequate specimen for histological examination in all cases and was not associated with any significant complication in this series. The frequency distribution of the various histological appearances is given in Table II.

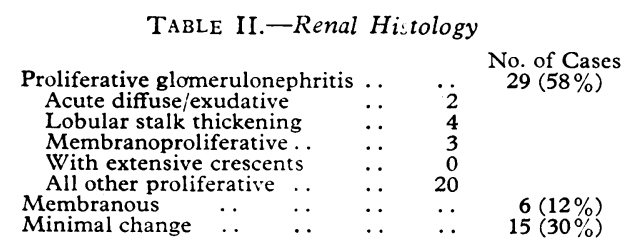

\section{Discussion}

We are unable to estimate how far our figure for the minimum incidence of the nephrotic syndrome falls short of the true incidence; but since the nephrotic syndrome in adults is now widely accepted as a strong indication for renal biopsy, and during the period of this study we were providing a routine renal biopsy service for most of the hospitals in the areas surveyed, it seems likely that a majority of suitable patients were included. Also it was hoped that if transfer of patients from their original hospitals could be avoided this would encourage the physicians of the region to allow inclusion of their patients. No figure is available in the literature for comparison with the minimum annual incidence obtained here of 9.0 per million adult population. Estimates are available, however, for the incidence in childhood. Rothenburg and Heymann (1957) made an estimate of an annual incidence of 23 per million for children aged $0-9$ in Ohio and 69 per million in Cleveland itself, but the latter figure was obtained by means of a postal questionary which relied on different paediatricians' opinions on whether the patients had a nephrotic syndrome, and may have overestimated the incidence of the condition as defined by strict criteria. Arneil (1961) suggested a figure of 20 per million for children aged 0-12 years in Scotland.

In the South-east and South-west Metropolitan Regions of England a collaborative trial of steroids in the nephrotic syndrome of childhood has been in progress since 1963 under the direction of Professor W. Holland of St. Thomas's Hospital. This trial, plus a search for other patients in the South-east Metropolitan Region (780,000 children), resulted in a figure of 17.5 new patients per million children aged $0-14$ per year for the five-year period 1963-8 (Cameron, White, and Ogg, unpublished). The same criteria as in the present survey were used for inclusion; if patients with a clinical diagnosis of Henoch-Schönlein purpura were included, the incidence rose to 21.4 . The present findings confirm that the nephrotic syndrome is less common in adults.

\section{Renal Histology}

In this series the relative frequency of the three main histological groups differs from that in most previously reported series (Robson, 1967) principally in the higher frequency of minimal change histology and the considerably lower frequency of membranous nephropathy. In view of the small numbers of patients chance variation is likely to have played a part, but other factors important in accounting for these differences include non-standard definition of the nephrotic syndrome, method of selection of patients for biopsy, and variation in interpretation of the histological appearances.

\section{Minimal Change Histology}

The $30 \%$ incidence of patients with minimal change histo$\operatorname{logy}$ in the survey is higher than that found in most other published series of adults (Robson, 1967). Only Berger et al. (1961) and McGovern (1964) found a higher proportion (44\% and $48 \%$ respectively), but the former included an unstated proportion of children. Since we attempted to minimize selection of patients in this series-for example, the self-exclusion by previous response to corticosteroids of the proportion of minimal change patients-we suggest that the figure of $30 \%$ may be a more realistic estimate of the frequency of minimal change histology in adult nephrotic patients.

The effect of selection before referral can be seen in our own data (Cameron, White, and Ogg, unpublished) for patients in the South-east Metropolitan Region:

$$
\begin{array}{cccccc}
\text { Percentage of Patients with } & \multicolumn{3}{c}{\text { Minimal Change Histology }} \\
\text { " Referred" } & & & \text { Adults } & \text { Children } \\
\text { "Unselected " } & \ldots & \ldots & 19 \% & 30 \% \\
& \ldots & \ldots & 30 \% & 78 \%
\end{array}
$$

the incidence in the "referred" patients being similar to that of most other series (Robson, 1967). The contrast with an incidence of $78 \%$ in a similarly investigated series of children (Cameron, White, and Ogg, unpublished) is striking, and accounts in a large measure for the poorer overall response to steroids of adults with the nephrotic syndrome (Cameron, 1968).

\section{Membranous Nephropathy}

The marked difference in the frequency of membranous nephropathy found here (12\%) and the mean figure of $38 \%$ in 14 previously published series (Robson, 1967) is likely to be largely due to the use of different histological criteria for this diagnosis.

Allocation to the group was made only if epimembranous deposits could be seen on the periodic acid-silver methenamine stained thin sections. It is now clear that a diagnosis of membranous nephropathy cannot be substantiated unless these deposits can be demonstrated by optical or electron microscopy. In the past, with the use of only haemalum-eosin or glycoprotein stains, it is likely that patients with membranoproliferative glomerulonephritis in whom the capillary wall thickening was prominent and the proliferation slight may have been allocated to the "membranous" group ; the distinction is clear if silver stains or electron microscopy are used. Further, glomerular scarring of ten leads to an apparent capillary wall thickening due to shrinkage and folding. Thick sections will also give the appearance of thickened capillary walls, especially if the endothelial cell cytoplasm is prominent.

If more lenient criteria for inclusion in the nephrotic group are taken, this also will enhance the apparent incidence of membranous nephropathy, since many patients with this condition have persistent proteinuria short of a full nephrotic syndrome. During the period of the trial a further five patients with membranous nephropathy were seen who had heavy proteinuria but who did not satisfy all the criteria for inclusion. 
Another suggestion to explain the discrepancy is that some patients in the present series with mild membranous nephropathy have been included in the minimal change group. This is very unlikely in view of the clinical presentation and course of the 15 patients allocated to the minimal change group. All had highly selective differential protein clearances (Cameron and Blandford, 1966) and normal renal function. Ten of these patients subscquently lost their proteinuria, either spontaneously or as the result of therapy in the associated trial (Sharpstone et al., 1969), and a further four showed great reduction in proteinuria with clinical improvement; only one patient failed to respond to steroids at all.

The possibility remains, however, that there may be geographical variations in the incidence of membranous nephropathy, and a number of clinicians have the impression from experience on either side of the Atlantic that the condition is commoner in the United States than in England.

\section{Sex Incidence}

Even allowing for the difficulty in assessing renal histology just discussed, the sex ratios found in this survey were surprising. In children, a group largely consisting of patients with minimal change histology, a rather constant male : female ratio of $2: 1$ is found (Rothenberg and Heymann, 1957; Arneil, 1961). Most previous series of adult nephrotics have shown either equal sex incidence or a preponderance of males (Heptinstall, 1966), though Pearl et al. (1963) also reported an excess of females in their series, which consisted of 53 adults and children.

\section{Renal Amyloidosis}

Although the trial protocol specifically excluded patients with evidence of multi-system disease, it is of interest that six patients $(10.7 \%$ of total) fulfilling the clinical and biochemical criteria for entty were found on renal biopsy to have amyloidosis. This is in line with the incidence of 11 to $12 \%$ in other series of nephrotic patients (Berman and Schreiner, 1958; Blainey et al., 1960 ; Maxwell et al., 1964) and emphasizes the value of renal biopsy in the nephrotic syndrome in adults. Only one patient had a possible associated condition (mild bronchiectasis), but during the period of the survey biopsy was carried out in a further three patients with amyloidosis, rheumatoid arthritis, and the nephrotic syndrome. This is in keeping with the finding of Brandt et al. (1968) of an equal incidence of the nephrotic syndrome in both primary and secondary amyloidosis.

This was a collaborative study, and the interest and work of large numbers of people made it possible. The consultant physicians of the South-east Metropolitan Region referred their patients to us and to them our thanks are due. Dr. J. S. S. Fairley of the Southeast Metropolitan Regional Hospital Board and his colleagues gave us every facility and a financial grant to help the survey. Dr. F. S. Goldby performed some of the biopsies, and Mr. J. Kaye and Mr. Gabriel Bankole carried out the biochemical estimations. The histological specimens were expertly processed by Mr. D. J. Ware, Mr. J. Osborne, and Mr. R. Francis.

\section{REFERENCES}

Arneil, G. C. (1961). Lancet, 2, 1103.

Berger, J., de Montera, H., and Galle, P. (1961). Archives d'Anatomie Pathologique, 9, 313.

Berman, L. B., and Schreiner, G. E. (1958). American fournal of Medicine, 24, 249.

Blainey, J. D., Brewer, D. B., Hardwicke, J., and Soothill, J. F. (1960). Quarterly fournal of Medicine, 29, 235.

Brandt, K., Cathcart, E. S., and Cohen, A. S. (1968). American Fournal of Medicine, 44, 955 .

Cameron, J. S. (1968). British Medical fournal, 4, 352.

Cameron, J. S. (1968). British Medical fournal, 4, 352.

Heptinstall, R. H. (1966). Pathology of the Kidney, p. 361. London. Hiller, A., McIntosh, J. F., and Van Slyke, D. D. (1927). Fournal of Clinical Investigation, 4, 235.

Kark, R. M., and Muehrcke, R. C. (1954). Lancet, 1, 1047.

McGovern, V. J. (1964). Australasian Annals of Medicine, 13, 306.

Maxwell, M. H., Adams, D. A., and Goldman, R. (1964). Annals of Internal Medictne, 60, 539.

Ogg, C. S., Cameron, J. S., and White, R. H. R. (1968). Lancet, 2, 78.

Pearl, M. A., Burch, R. R., Carvajal, E., McCracken, B. H., Woody, H. B., and Sternberg, W. H. (1963). Archives of Internal Medicine, $112,716$.

Robson, J. S. (1967). In Renal Disease, 2nd ed., edited by D. A. K. Black, p. 276. Oxford.

Rothenberg, M. B., and Heymann, W. (1957). Pediatrics, 19, 446.

Sharpstone, P., Ogg, C. S., and Cameron, J. S. (1969). British Medical Fournal, 2, 535 .

\title{
Nephrotic Syndrome Due to Primary Renal Disease in Adults: II. A Controlled Trial of Prednisolone and Azathioprine
}

\author{
P. SHARPSTONE,* M.B., M.R.C.P.; C. S. OGG, † M.D., B.SC., M.R.C.P. ; J. S. CAMERON, $\ddagger$ M.D., B.SC., M.R.C.P.
}

\begin{abstract}
Cummary : A controlled trial in 20 adult patients with $\checkmark$ the nephrotic syndrome due to proliferative glomerulonephritis compared the effects of consecutive eight-week courses of treatment with prednisolone in conventional dosage and a low-dose azathioprine-prednisolone combination. Though the azathioprine regimen avoided serious toxicity and produced a statistically significant improvement in creatinine clearance and urine protein excretion, the results were not significantly better than with prednisolone itself and overall were not of great clinical value. Prolongation of the azathioprine-predniso-
\end{abstract} * Lecturer in Medicine, King's College Hospital Medical School, London
S.E.5.

† Renal Physician, Guy's Hospital, London S.E.1.

₹ Renal Physician and Senior Lecturer, Department of Medicine, Guy's Hospital, London S.E.1. lone treatment to one year was associated with some small improvement in the results.

The effectiveness of prednisolone in the nephrotic syndrome of adults with minimal renal histological abnormality was confirmed by a complete loss of proteinuria within eight weeks in six out of eight patients and substantial reduction in the other two. These two patients subsequently received azathioprine as well but with equivocal results.

\section{Introduction}

The value of corticosteroids in the treatment of adult patients with the nephrotic syndrome is small because this is largely confined to those with mild or minimal lesions of the glomeruli 